\title{
Multi-projector three-dimensional display for 3D Geographic Information System
}

\author{
Shujun Xing ${ }^{1}$, Shuo Liu ${ }^{* 2}$ Xinzhu Sang ${ }^{1}$ \\ ${ }^{1}$ State Key Laboratory of Information Photonics and Optical Communications, Beijing University of Posts and \\ Telecommunications (BUPT), 100876 Beijing, China \\ ${ }^{2}$ Key Laboratory of Digital Earth Science, Institute of Remote Sensing and Digital Earth, Chinese Academy of Sciences, \\ Beijing 100094, China \\ Corresponding author's email: *liushuo@ radi.ac.cn
}

\begin{abstract}
A three-dimensional display system to show a given three-dimensional Geographic Information System (GIS) scene is presented, which is based on a digital light projector array, common PC clients, a projection diffusion screen augmented with a micro-lens array. A solution to calibrate the dynamic projectors' image and an efficient method to render and synchronize view-point images via a cluster of PC clients are proposed. The method to evaluate the distortion of GIS in the system is discussed. Dynamic 3D GIS scenes with stereo parallax and smooth parallax are demonstrated.
\end{abstract}

Key words: Multi-projector 3D display; GIS; distortion; synchronization

\section{Introduction}

In recent years, the development of Geographic Information System （GIS ） has dedicated to big data processing and visualization of geographic information. With the increasing of the data volume, it is more and more difficult to express the comprehensive information by $2 \mathrm{D}$ display device for the use of 3D GIS. The 3D display technology has found many applications, such as medical treatment[1][2],urban environments[3],maps[4][5],video telephony[6],advertise[13] and so on. However, the large-size 3D display screen is required to meet the demand of the 3D GIS display. Recently, a large size and high dense views three-dimensional display based on frontal projection with a LLA and a diffusion screen is demonstrated in our laboratory [7, 8]. Here, the glasses-free 3D display system is improved to show the 3D GIS scene, such as battlefield scene, smart city scene or wisdom agriculture scene. It can easily help viewers to understand the useful 3D GIS information and enhance human object location Memory[5].Three problems for constructing the GIS 3D display system is the focus to discuss:(1) how to render 3D image synchronously, (2)how to calibrate each projector image for multi-view 3D screen and (3)how to evaluate the distortion of Geo-spatial information.

\section{System hardware structure and configuration}

As shown in Fig.1, the GIS 3D projection system contains one sever, 24 PC clients, 24 micro projectors and a $1.9 \mathrm{~m} \times 1.2 \mathrm{~m} 3 \mathrm{D}$ diffusion screen. The server sends its virtual camera parameters 
in 3D GIS system to each PC client which adjusts its virtual camera parameters according to the server's commands. Each PC client renders its viewpoint image and calibrates the image for the multi-view 3D diffusion screen. The view zone distribution of the screen is shown in Fig.2. The CPU used in PC clients and sever is Intel(R) Core(TM) I5-4460 and the RAM memory capacity is 8 GB. The total band width of network for PC clients and server is 1 Gbps. Configurations of projectors and screen is same as the Reference [7].

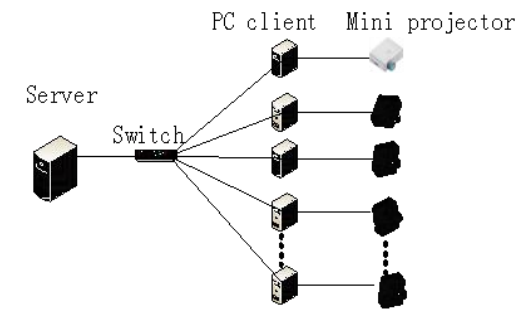

(a)



(b)

Fig.1 Structure of the display system:

(a) Structure diagram of the display system

(b)the running effect of the 3D display system.

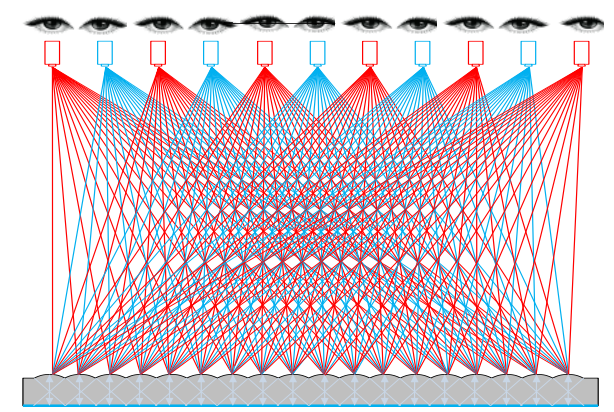

Fig.2 The diagram of Multi-view projection screen and its view zone distribution

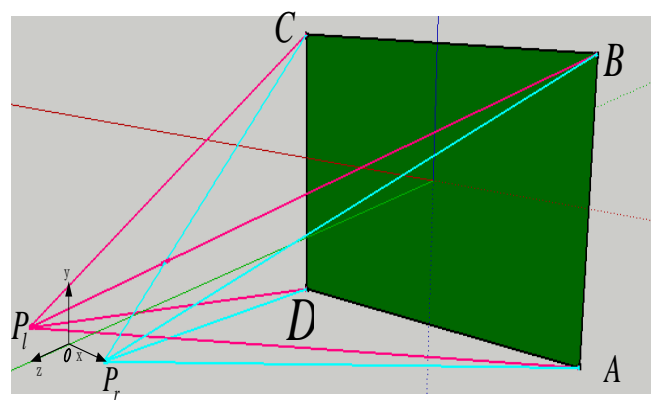

Fig.3 The frustum of eyes : Plane $A B C D$ represents screen,$P_{l} P_{r}$ represents the position of left eye and right eye

\section{Multi-View Rendering and synchronization}

\section{1 Multi-view image rendering method}

Different projectors should project different viewpoint images to produce multi-view 3D image. The viewpoint image rendered by the 3D engine is determined by the virtual camera's view matrix and perspective matrix. As shown in Fig.3, $P_{l}\left(e_{l x}, e_{l y}, 0\right)$ and $P_{r}\left(e_{r x}, e_{r y}, 0\right)$ represent 
positions of the viewer's left eye and right eye. The virtual camera view frustum $P_{l}^{\prime} A^{\prime} B^{\prime} C^{\prime} D^{\prime}$ and $P_{r}^{\prime} A^{\prime} B^{\prime} C^{\prime} D^{\prime}$ in the virtual world should match with the view frustum $P_{l} A B C D$ and $P_{r} A B C D$ in the real world, respectively. The virtual camera's settings should meet the Eq. (1) $[8]$,

$$
\frac{\overrightarrow{O A}}{\overrightarrow{O_{v} A_{v}}}=\frac{\overrightarrow{O B}}{\overrightarrow{O_{v} B_{v}}}=\frac{\overrightarrow{O C}}{\overrightarrow{O_{v} C_{v}}}=\frac{\overrightarrow{O D}}{\overrightarrow{O_{v} D_{v}}}=\frac{\overrightarrow{O P_{l}}}{\overrightarrow{O_{v} P_{l v}}}=\frac{\overrightarrow{O P_{r}}}{\overrightarrow{O_{v} P_{r v}}}=1
$$

Here, $\mathrm{O}$ is the position of one eye. Given that the distance from point $O$ to plane $A B C D$ is $D_{s}$, the view matrix of symmetric view frustum $O^{\prime} A^{\prime} B^{\prime} C^{\prime} D^{\prime}$ is $M_{v}$ and the perspective matrix of view frustum $O^{\prime} A^{\prime} B^{\prime} C^{\prime} D^{\prime}$ is $M_{p} . M_{v p}$ is the matrix of viewport transform and the world coordinate of point $p$ in virtual world is $(x, y, z, 1)$. In computer graphics, the coordinate of point $p$ in the 2D image rendered via the virtual camera $O^{\prime} A^{\prime} B^{\prime} C^{\prime} D^{\prime} \quad$ is $p \bullet M_{v} \bullet M_{p} \bullet M_{v p}$.

According to the above constraints, we get the coordinate of point $p$ in 2D image that rendered by the virtual camera $P_{l}^{\prime} A^{\prime} B^{\prime} C^{\prime} D^{\prime} \quad$ through the following Eq. (2):

$$
P_{l}=p \bullet V \bullet M_{v} \bullet M_{t} \bullet M_{\text {shear }} \bullet M_{p} \bullet M_{v p}
$$

$$
\text { where } M_{t}=\left(\begin{array}{cccc}
1 & 0 & 0 & 0 \\
0 & 1 & 0 & 0 \\
0 & 0 & 1 & 0 \\
e_{l x} & e_{l y} & 0 & 1
\end{array}\right), M_{\text {shear }}=\left(\begin{array}{cccc}
1 & 0 & 0 & 0 \\
0 & 1 & 0 & 0 \\
e_{l x} / D_{v s} & e_{l y} / D_{v s} & 1 & 0 \\
0 & 0 & 0 & 1
\end{array}\right) \text {. }
$$

Thus, the asymmetric view frustum to get viewpoint image can be deduced from the symmetric view frustum. The new viewpoint virtual camera' view matrix is $M_{v} \bullet M_{t}$ and the new perspective matrix is $M_{\text {shear }} \bullet M_{p}$. The multi-view virtual cameras can be easily set by the Eq. (2), which is adapted to the horizontal parallax and the full parallax situation. There are 24 view-zones in our system, and each view-zone virtual camera share the $O^{\prime} A^{\prime} B^{\prime} C^{\prime} D^{\prime}$ virtual camera's view matrix and perspective matrix via local area network. When the 3D GIS scene changes, the 3D GIS sever sends the view matrix and perspective matrix of $O^{\prime} A^{\prime} B^{\prime} C^{\prime} D^{\prime}$ virtual camera to every PC client. 


\section{2 The synchronization between sever and PC clients}

In the distributed system, the PCs' hardware may be different from each other, and the rendering time of every viewpoint image is also different. When the rendering time interval between viewpoint images is more than a certain value, the viewer may observe totally different images for both eyes at the same time, which is unacceptable. Different 3D GIS scenes should be separately synchronized in two situations. The first situation is that the 3D GIS scene only contains Raster data-satellite image data, elevation data and vector data. The second situation is the mobile model in the 3D GIS scene.

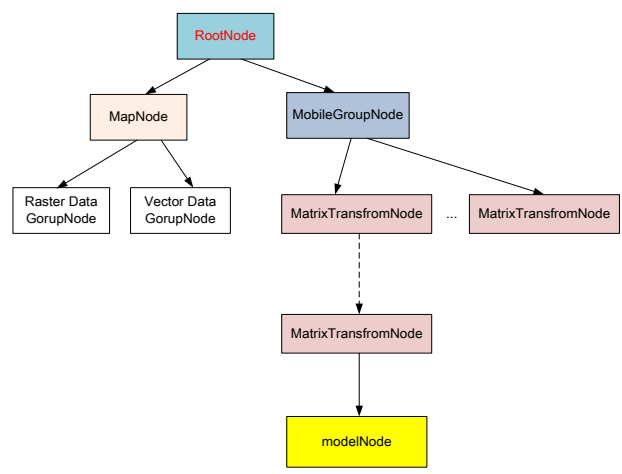

Fig.4 Scene graph in 3D GIS

In the system, OSGEarth 3D terrain engine is used to design a scene graph which is data structure used to organize a scene in the 3D GIS system. Scene graph is a collection of nodes in a tree or graph structure. In our system as shown in Fig.4, it only have one root node and the root node have two child nodes-MapNode and MobileGroupNode. MapNode manages GIS raster data and vector data which is always static and easily process by the levels of detail (LOD) technology. MobileGroupNode is used to manage mobile object in Geographical space, such as air-plane ,boat, truck etc.

\subsection{Raster data and vector data synchronization}

LOD technology is applied in 3D GIS to load raster data and vector data quickly. Firstly, the raster data is converted to tile map format which follows Bing maps conventions. Secondly, PC client uses OSGEarth engine to load the tile map and render itself viewpoint image. PC clients maybe render different level raster data because of different virtual camera positions. However, only the resolution of different level raster image for same area is different, and the viewer is hard to detect the difference of different viewpoints with different zoom levels as shown in Fig.5. 


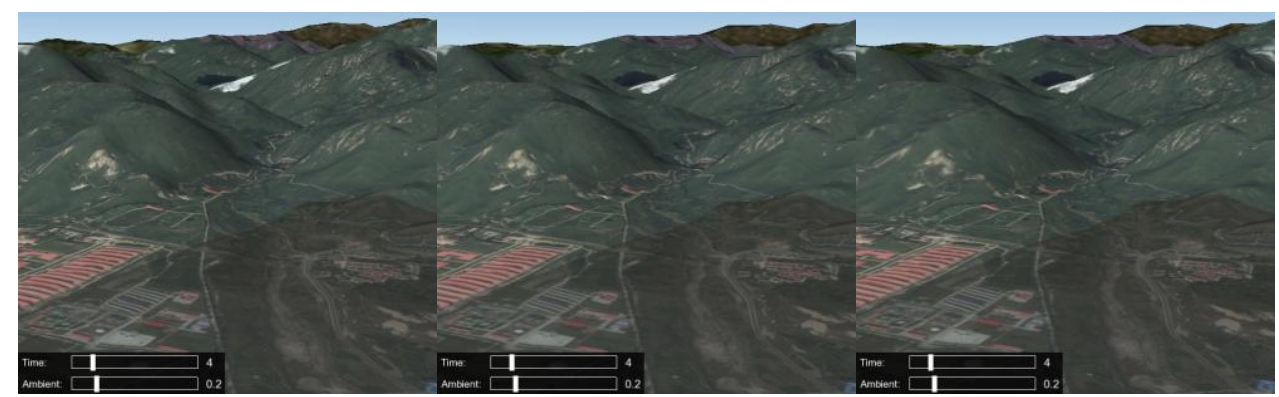

Fig.5(a) zoom level 18

(b) zoom level 17

(c) zoom level 16

From the above analysis, when the 3D GIS scene only contains raster data and vector data, the virtual camera's synchronization should be considered. Because the data size of the model-view matrix and projection matrix is 256 bytes, the delay time caused by network transmission can be ignored. Assuming that the frame rate of 3D GIS system is $60 \mathrm{fps}$, the date size sent by network UDP broadcast is 15360 bytes in one second. It only accounts for $0.01 \%$ of the total band width 1.0 Gbps. The time sync mechanism for network is excluded from consideration. PC client is only used to read the newest shared view matrix and projection matrix for rendering the new frame of 3D GIS scene.

\subsubsection{Mobile model synchronization in 3D GIS scene}

Many mobile objects are considered in the 3D GIS scene, such as airplanes moving in different places in the 3D GIS system. As shown in Fig.6 and Fig.7, every point in 3D GIS space has its local geographic Cartesian coordinate frame and its transformation matrix is $M_{G L}$ relative to the world Cartesian coordinate frame. The $\mathrm{X}$ axis point to the east, the $\mathrm{Y}$ axis point to the north, the $\mathrm{Z}$ axis is parallel with the line form the earth center to the original point of the coordinate frame. Each plane has its own local coordinate frame kmn relative to local Geographic Cartesian coordinate frame $x y z$ and its transformation matrix is $M_{B L}$. The propeller local coordinate frame $p q r$ is relative to local plane Cartesian coordinate frame $k m n$ and its transformation matrix is $M_{S L}$. The transformation matrix form propeller local coordinate frame to world coordinate frame is $M_{G L} * M_{B L} * M_{S L}$. In OSGEarth, each transform matrix corresponds to a matrix transform node. 


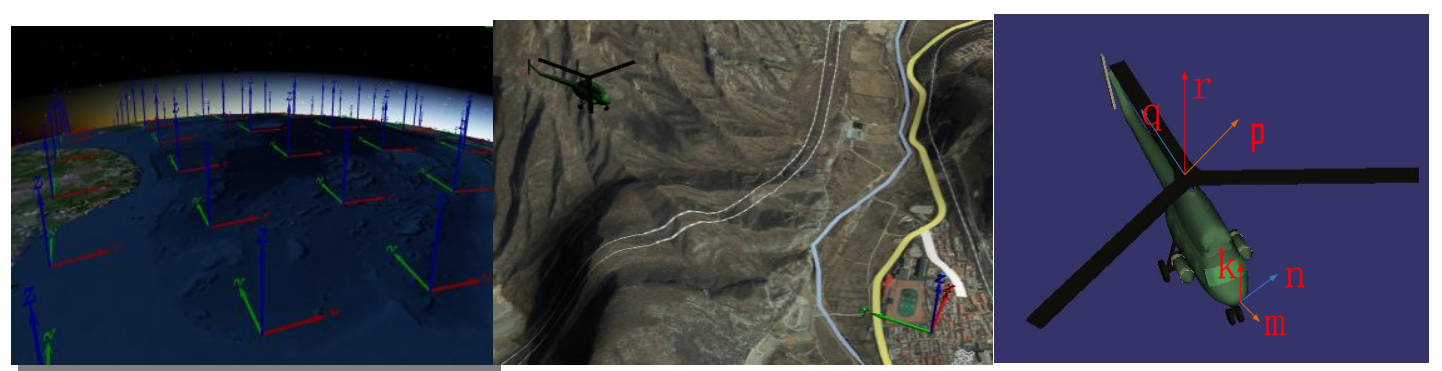

Fig.6 Mobile model in 3D GIS scene

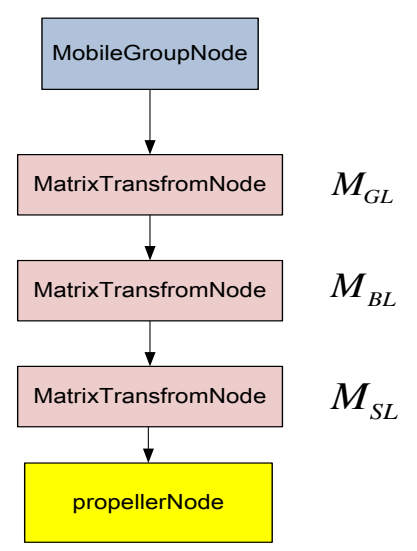

Fig.7 The relationship of transform matrix nodes

Sever sends the entire changed local transform matrix and its node name to PC clients under current view frustum. The changed local transform matrix is sorted by its name, and the sort method is binary insertion sort. PC clients set the changed local transform matrix to the corresponding transform matrix node by searching the node name.

\section{Display Calibration}

The system contains 24 micro projectors for projecting different viewpoint images from different positions to the front-projection lenticular diffusing screen. As we known, unless the projector's optical axis is carefully aligned to be perpendicular to the screen, the projected image appears distorted due to the perspective effect, which greatly constrains the placement of the projector for the Multi-projector auto-stereoscopic display. As shown in the Fig.8, projector A is placed at the right position and obtains the right viewpoint image, but other projector's viewpoint images have various degrees of distortion. Projector B is in the inapposite position and its image appears as irregular quadrilateral. Virtual camera is an ideal pin-hole camera in computer graphics, and projectors can be treated as pin-hole cameras. If source images of projector $\mathrm{A}$ and $\mathrm{B}$ come from the same virtual camera, we can show that the compounded transforms mapping projector $\mathrm{A}$ point $(x ; y)$ to projector B point $(X, Y)$ in the XOY plane, which can be expressed by a single projection transformation, 


$$
(x, y)=\left(\frac{h_{1} X+h_{2} Y+h_{3}}{h_{7} X+h_{8} Y+1}, \frac{h_{4} X+h_{5} Y+h_{6}}{h_{7} X+h_{8} Y+1}\right)
$$

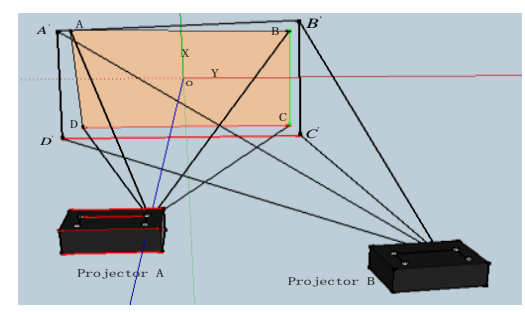

Fig.8 Image distortion caused by the micro projector

The calibration parameters of projector B can be computed by four control point pairs, $\left(A, A^{\prime}\right),\left(B, B^{\prime}\right),\left(C, C^{\prime}\right)$ and (D,D'). Since the image pixel process for calibration by CPU is time-consuming, we write a GLSL script to execute the method in GPU. OpenGL renders the 3D GIS data to the FBO(frame buffer object ), and GPU reads the FBO as a texture for calibration and then outputs the final image to screen.

\section{3D Geo-spatial information distortion analysis in the system}

From the references [10] [11], we can know distortions are caused by differences in photographing, projection conditions and viewers' position. According to the reference [8], the condition without distortion for 3D images should meet the Eq. (1). Here, the distortion influence on geo-spatial information comprehension is discussed.
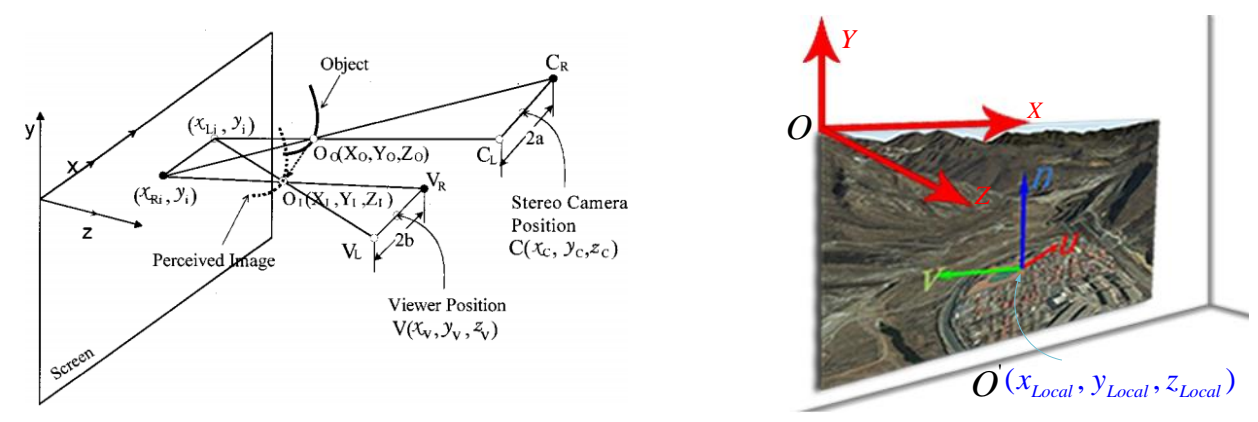

Fig.9 (a) Coordinates for stereo image calculation.

(b) the local geographic Coordinate frame $u v n$ and the screen coordinate frame $X Y Z$

$\left(X_{I}, Y_{I}, Z_{I}\right)$ in Fig.9 $(\boldsymbol{a})$ represents a point object that viewers can perceive in the screen [11]. 
In the Cartesian coordinate $u v n$ centered at the position $O^{\prime}$ (its height above sea level is zero), as shown in Fig.9(b), when the plane $v O^{\prime} \mathrm{n}$ is parallel with the sea level, the perceived point object $\left(u_{o}, v_{o}, n_{o}, 1\right)$ shown in 3D GIS space is as $\left(u_{I}, v_{I}, n_{I}, 1\right)$ which can be expressed as the following equation.

$$
\begin{gathered}
\left(u_{t}, v_{l}, n_{t}, 1\right)=\left(u_{o}, v_{o}, n_{o}, 1\right)\left[\begin{array}{cccc}
x_{u} & x_{v} & x_{n} & 0 \\
y_{u} & y_{v} & y_{n} & 0 \\
z_{u} & z_{v} & z_{n} & 0 \\
-x_{\text {Local }} & -y_{\text {Local }} & -z_{\text {Local }} & 1
\end{array}\right]\left(\frac{2}{\left(2 b+x_{L i}-x_{R i}\right)\left(Z_{c}-Z_{o}\right)}\right)\left[\begin{array}{cccc}
b z_{c} & 0 & 0 & 0 \\
a x_{v}-b x_{c} & b z_{c} & 0 & 0 \\
a y_{v}-b y_{c} & 0 & a z_{v} & 0 \\
0 & 0 & 0 & 1
\end{array}\right]\left[\begin{array}{cccc}
x_{u} & y_{u} & z_{u} & 0 \\
x_{v} & y_{v} & z_{v} & 0 \\
x_{n} & y_{n} & z_{n} & 0 \\
e & f & g & 1
\end{array}\right](5) \\
\text { Here, } e=\left|\begin{array}{lll}
x_{\text {local }} & y_{u} & z_{u} \\
y_{\text {local }} & y_{v} & z_{v} \\
z_{\text {local }} & y_{n} & z_{n}
\end{array}\right|, f=\left|\begin{array}{lll}
x_{u} & x_{\text {Local }} & z_{u} \\
x_{v} & y_{\text {Local }} & z_{v} \\
x_{n} & z_{\text {Local }} & z_{n}
\end{array}\right|, g=\left|\begin{array}{lll}
x_{u} & y_{u} & x_{\text {local }} \\
x_{v} & y_{v} & y_{\text {local }} \\
x_{n} & y_{n} & z_{\text {local }}
\end{array}\right|,
\end{gathered}
$$

So $n_{I}$ represents the height above sea level of the perceived point object.

In most situation, since $\overrightarrow{V_{R} V_{L}} \bullet \overrightarrow{O^{\prime} \mathrm{n}}=0$, the Eq. (5) is simplified as

$$
\left(u_{l}, v_{l}, n_{l}, 1\right)=\left(u_{o}, v_{o}, n_{o}, 1\right) R_{Z} R_{X} T\left(\frac{2}{\left(2 b+x_{L i}-x_{R i}\right)\left(Z_{c}-Z_{o}\right)}\right)\left[\begin{array}{cccc}
b z_{C} & 0 & 0 & 0 \\
a x_{v}-b x_{c} & b z_{C} & 0 & 0 \\
a y_{v}-b y_{c} & 0 & a z_{v} & 0 \\
0 & 0 & 0 & 1
\end{array}\right]\left(R_{Z} R_{X} T\right)^{-1}
$$

where $R_{Z}, R_{X}$ are Z-axis Rotation matrix and $\mathrm{X}$-axis Rotation matrix, respectively.

\section{1 Distortions by Differences in parameters a, b, V, C}

When $R_{Z} R_{X} T$ is equal to the identity matrix, viewers' look up vector is perpendicular to horizontal plane and the angle of rotation matrix is 0 .

5.1.1 Assuming that $\mathrm{V}=\mathrm{C}$ and $b=63 \mathrm{~mm}$ [12]in Fig.9(a), the center of an $10 \times 10$ rectangle is positioned on $(0,0,100)$ in the GIS space. The height and area of rectangle can be impacted by the distance $b$ as is depicted in Fig.10 (a). For the case of $a>b$, the depth of the perceived rectangle becomes bigger and the area becomes smaller. From the Fig.10 (b), we can know that with the increase of $b$, the perceived depth of the rectangle becomes greater with the style of nonlinear growth . So the height of terrain and the area of horizontal profile chart are affected by the distance between virtual cameras. 

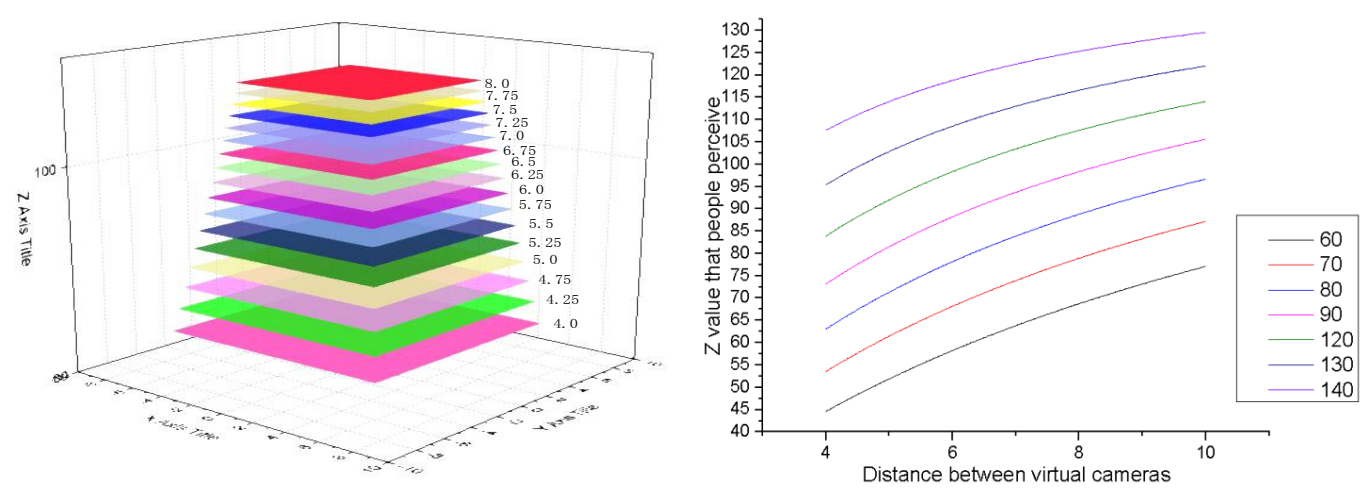

Fig. 10(a) the rectangle perceived by viewers with different value of $\boldsymbol{b}$

(b) The perceived depth of many rectangles with different position by setting different distances between virtual cameras

5.1.2 Assuming that $\boldsymbol{a}=63 \mathrm{~mm}[12], x_{c}$ is set to different values and other conditions are same as the situation (5.1.1). Fig.11 (a) shows that with the increase of $\Delta x$, the $X$ value of the rectangle becomes smaller and its $Z$ value keeps unchanged. For instance, given that $\Delta x=5$ and five different $10 \times 10$ rectangles paralleling to the XOY plane, their centers are $(0,0,80),(0,0,90)$, $(0,0,100),(0,0,110),(0,0,120)$ respectively in Fig.11(b). When $\mathrm{z}$ increases, the distortions at $\mathrm{X}$ axis become bigger. Under this situation, $x_{c}$ only influences the slope of terrain, and the height of terrain is not influenced.
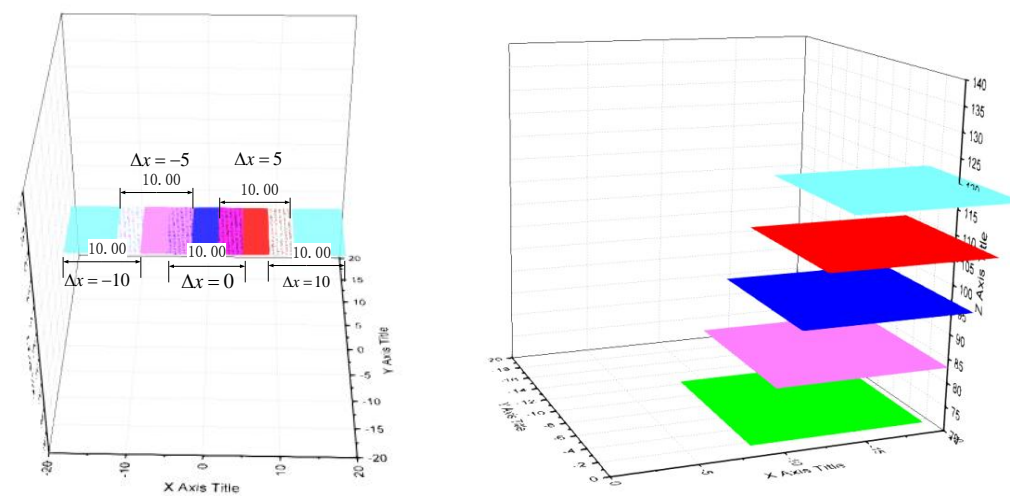

Fig.11 (a) $x_{c}$ is set to different values, and other parameters keep unchanged, $\Delta x=x_{c}-x_{v}$

(b) Five rectangles with different $\mathrm{z}$ values for the $\Delta x=5$ case.

\section{2 The GIS spatial information distortions caused by the} parameter $R_{z}$

Setting $R_{Y} T$ to the identity matrix, so viewer's look up vector is perpendicular to the horizontal 
plane. The angle of rotation matrix $R_{z}$ is $\gamma$, so $R_{z}=\left[\begin{array}{cccc}\cos r & \sin r & 0 & 0 \\ -\sin r & \cos r & 0 & 0 \\ 0 & 0 & 1 & 0 \\ 0 & 0 & 0 & 1\end{array}\right]$

The position $\left(u_{I}, v_{I}, n_{I}, 1\right)$ can be expressed as

$$
\left(u_{I}, v_{I}, n_{I}, 1\right)=\left(\begin{array}{l}
\frac{b z_{c} u_{o}+n_{o}\left(a X_{v}-b X_{c}\right) \cos \gamma+n_{o}\left(a y_{v}-b y_{c}\right) \sin \gamma}{b z_{c}+n_{o}(a-b)} \\
\frac{b z_{c} v_{o}-n_{o}\left(a X_{v}-b X_{c}\right) \sin \gamma+n_{o}\left(a y_{v}-b y_{c}\right) \cos \gamma}{b z_{c}+n_{o}(a-b)} \\
\frac{a n_{o} z_{v}}{b z_{c}+n_{o}(a-b)}, \\
1
\end{array}\right)
$$

So the height of terrain $\mathrm{O}$ is not affected by the angle $\gamma$.

If $\mathrm{a}=\mathrm{b}$ and $y_{v}=y_{c}$, the Eq. (7) can be further simplified as

$$
\left(u_{I}, v_{I}, n_{I}, 1\right)=\left(u_{o}+\frac{n_{o}}{z_{c}}\left(x_{v}-x_{c}\right) \cos r, v_{o}-\frac{n_{o}}{z_{c}}\left(x_{v}-x_{c}\right) \sin r, n_{o}, 1\right)
$$

The results in the following figure can be achieved.

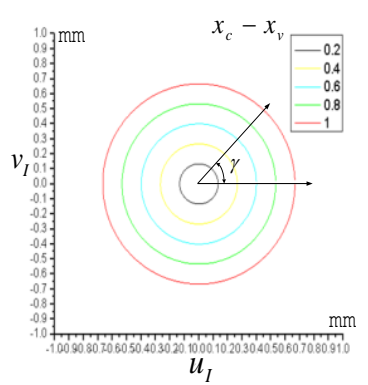

Fig.12 The point $(0,0,100)$ perceived by viewer, when $\left(x_{c}-x_{v}\right)$ and $\gamma$ is set to different value, $\gamma \in[0,2 \pi)$

\section{3 The GIS spatial information distortions caused by the parameter $R_{x}$}

If $R_{Z} T$ is the identity matrix, $R_{X}=\left[\begin{array}{cccc}1 & 0 & 0 & 0 \\ 0 & \cos \alpha & \sin \alpha & 0 \\ 0 & -\sin \alpha & \cos \alpha & 0 \\ 0 & 0 & 0 & 1\end{array}\right]$, the angle of the rotation matrix 
$R_{X}$ is $\alpha$, and then $\left(u_{I}, v_{I}, n_{I}, 1\right)$ can be expressed as

$\left(u_{l}, v_{l}, n_{l}, 1\right)=\frac{1}{b z_{c}+\left(v_{o} \sin \alpha+n_{o} \cos \alpha\right)(a-b)} \cdot\left(\begin{array}{l}b u_{o} z_{c}+\left(v_{o} \sin \alpha+n_{o} \cos \alpha\right)\left(a x_{v}-b x_{c}\right), \\ \left(b\left(v_{o} \cos \alpha-n_{o} \sin \alpha\right) z_{c}+\left(n_{o} \cos \alpha+v_{o} \sin \alpha\right)\left(a y_{v}-b y_{c}\right)\right) \cos \alpha+a z_{v}\left(v_{o} \sin \alpha+n_{o} \cos \alpha\right) \sin \alpha, \\ -\left(b\left(v_{o} \cos \alpha-n_{o} \sin \alpha\right) z_{c}+\left(n_{o} \cos \alpha+v_{o} \sin \alpha\right)\left(a y_{v}-b y_{c}\right)\right) \sin \alpha+a z_{v}\left(v_{o} \sin \alpha+n_{o} \cos \alpha\right) \cos \alpha, \\ 1\end{array}\right)$

The Eq. (8) illustrates that the position and the height of object in Geo-space are influenced by the angle of rotation $\alpha$. When $\alpha=\pi / 2$, the Eq. is

$$
\left(u_{I}, v_{I}, n_{I}, 1\right)=\frac{1}{b z_{c}+v_{o}(a-b)} \bullet\left(\begin{array}{l}
b u_{o} z_{c}+v_{o}\left(a x_{v}-b x_{c}\right) \\
a z_{v} v_{o} \\
b n_{o} z_{c}-v_{o}\left(a y_{v}-b y_{c}\right) \\
1
\end{array}\right)
$$

The above equation means that viewers' look up vector parallels to the horizontal plane, and parameters a and $\mathrm{b}$ will impact the position and height. The value of $u_{I}$ is influenced by the parameters $x_{c}, x_{v}$, and the parameter $y_{c}, y_{v}$ only have influences on the value of $n_{I}$.

\section{4 The GIS spatial information distortion caused by the}

\section{parameter $\mathrm{T}$}

When $R_{Z} R_{X}$ is equal to the identity matrix , the translate vector is $(m, n, k)$, and the perceived position is

$$
\left(u_{I}, v_{I}, n_{I}, 1\right)=\left[\begin{array}{c}
\frac{\left(b\left(u_{o}+m\right) z_{c}+\left(n_{o}+\mathrm{k}\right)\left(a x_{v}-b x_{c}\right)\right)}{b z_{c}+\left(n_{o}+k\right)(a-b)}-m \\
\frac{\left(b\left(v_{o}+n\right) z_{c}+\left(n_{o}+\mathrm{k}\right)\left(a y_{v}-b y_{c}\right)\right)}{b z_{c}+\left(n_{o}+k\right)(a-b)}-n \\
\frac{\left(a\left(n_{o}+\mathrm{k}\right) z_{v}\right)}{b z_{c}+\left(n_{o}+k\right)(a-b)}-k \\
1
\end{array}\right]
$$

The Eq. (9) illustrates that the value of $\mathrm{k}$ only can impact the value of $u_{I}, n_{I}$, the value of $u_{I}$ is affected by $\mathrm{m}$, and $\mathrm{n}$ only affect the value of $n_{I}$. 


\section{5 The distortion factor definition}

From the analyses above, we can know that when $\mathrm{a}=\mathrm{b}$ and $\mathrm{C}(x, y, z)=V(x, y, z)$, the distortions of 3D GIS information doesn't exist. Otherwise, the distortion of 3D GIS information is caused by parameter a, b, $\mathrm{C}(x, y, z), V(x, y, z)$ and $R_{Z} R_{X} T$.

A factor is defined to measure the range of distortion.

$$
D=|\log (b / a)|+|\overrightarrow{C V}| / Z_{c}
$$

When a $=\mathrm{b}=63 \mathrm{~mm}$, the factor can be simplified as $D=|\overrightarrow{C V}| / Z_{c}$

\section{Results and Discussions}

\section{1 The result of the rendering method}

According to the Eq. (2), every viewpoint image can be rendered by PC clients via different model-view matrix and projection matrix settings. Different viewpoint images are shown in Fig.13.

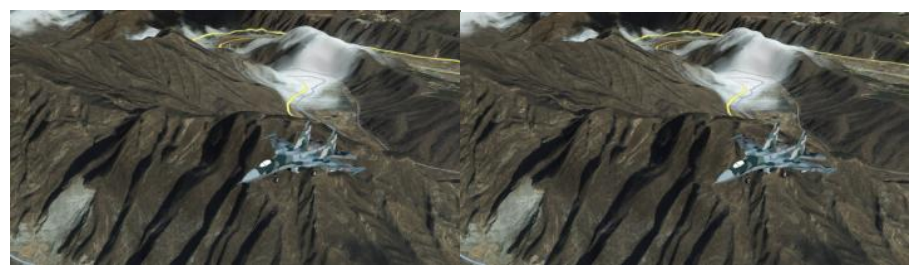

(a) The first viewpoint image

(b) The eight viewpoint image

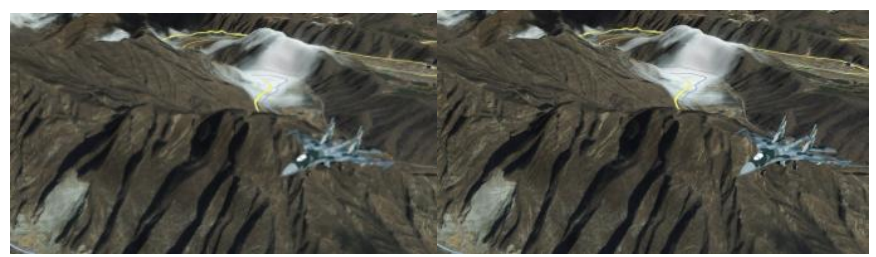

(c) The sixteenth viewpoint image

(d) The twenty-fourth viewpoint image

Fig.13 Different viewpoint images rendered by different PC clients 


\section{2 The result of calibration for multi-viewpoint images}

According to the Eq. (4), two kinds of algorithm are designed to test the running time. One is based on traditional CPU, and another one is based on the GPU. Table. 1 shows that the running time of traditional CPU calibration algorithm for one frame image is proportional to its image resolution ratio. The calibration algorithm based on GPU is not affect by the resolution ratio if the graphic card memory capacity for texture is large enough. The final calibration effect is shown in the Fig.14.

\begin{tabular}{|c|c|c|}
\hline $\begin{array}{c}\text { Image resolution } \\
\text { (format BMP 24bit) }\end{array}$ & CPU & GPU \\
\hline $640 \times 480$ & $9 \mathrm{~ms}$ & $4 \mathrm{~ms}$ \\
\hline $800 \times 600$ & $14 \mathrm{~ms}$ & $4 \mathrm{~ms}$ \\
\hline $1024 \times 768$ & $26 \mathrm{~ms}$ & $4 \mathrm{~ms}$ \\
\hline $1440 \times 900$ & $46 \mathrm{~ms}$ & $4 \mathrm{~ms}$ \\
\hline $1920 \times 1080$ & $69 \mathrm{~ms}$ & $4 \mathrm{~ms}$ \\
\hline
\end{tabular}

Table.1 The consuming time with different processing algorithms

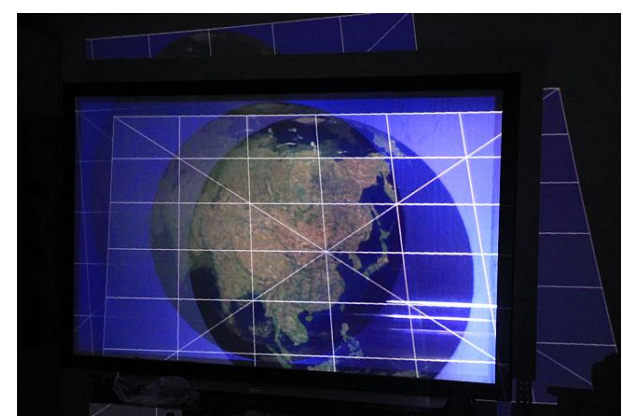

(a)

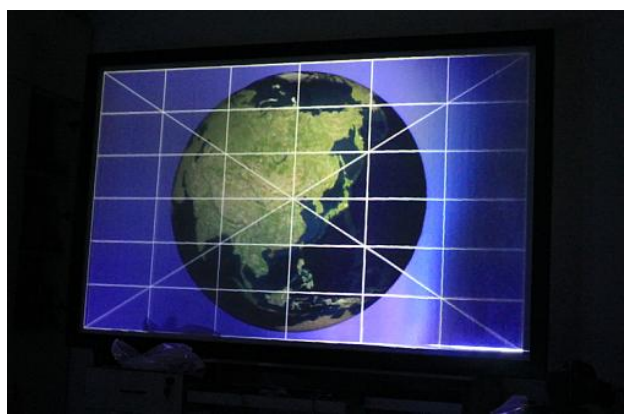

(b)

Fig.14 The result of calibration for projectors:

(a) the image before calibration(b) the image after calibration

\section{3 The result of the 3D GIS scene synchronization}

The Table 2 and Fig.15 show the average PC and server consuming time to synchronize the system with different amounts of mobile objects in the experiment. 


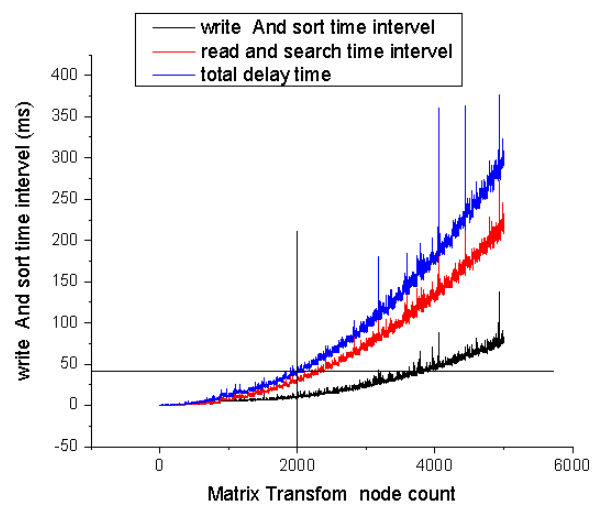

Fig.15 The consuming time for synchronization

Table 2 The consuming time for different numbers of mobile objects

\begin{tabular}{|c|c|c|c|}
\hline $\begin{array}{c}\text { Matrix } \\
\text { Transform } \\
\text { Node Count }\end{array}$ & $\begin{array}{c}\text { sever } \\
\text { consume } \\
\text { time (ms) }\end{array}$ & $\begin{array}{c}\text { PC consume } \\
\text { time(ms) }\end{array}$ & $\begin{array}{c}\text { Total delay } \\
\text { time (ms) }\end{array}$ \\
\hline 500 & 1 & 2 & 3 \\
\hline 1000 & 5 & 7 & 12 \\
\hline 1500 & 7 & 17 & 24 \\
\hline 2000 & 10 & 31 & 41 \\
\hline 2500 & 15 & 47 & 62 \\
\hline 3000 & 22 & 82 & 104 \\
\hline 3500 & 33 & 98 & 131 \\
\hline 4000 & 45 & 131 & 176 \\
\hline 4500 & 63 & 171 & 234 \\
\hline
\end{tabular}

As we set the frame rate $25 \mathrm{fps}$, the count of synchronization nodes should be less than 2000 matrix nodes in the view frustum.

\section{4 The result of 3D Geo-spatial information distortion and discussion}

Form the Eq. (5) and distortion analysis, the Table.3 is summarized to illustrate the influence of the parameters on 3D Geo-spatial point when viewers' interpupillary distance is equal to the distance between two virtual cameras.

Table.3 The influence of parameters on the position $\left(u_{I}, v_{I}, n_{I}\right)$ when $\mathrm{a}=\mathrm{b}$. 
“ " represents the parameter changing or the position distorting.

“-” represents that parameter meet the Eq. (1) or the position is no distortion.

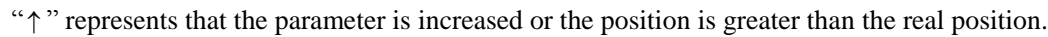

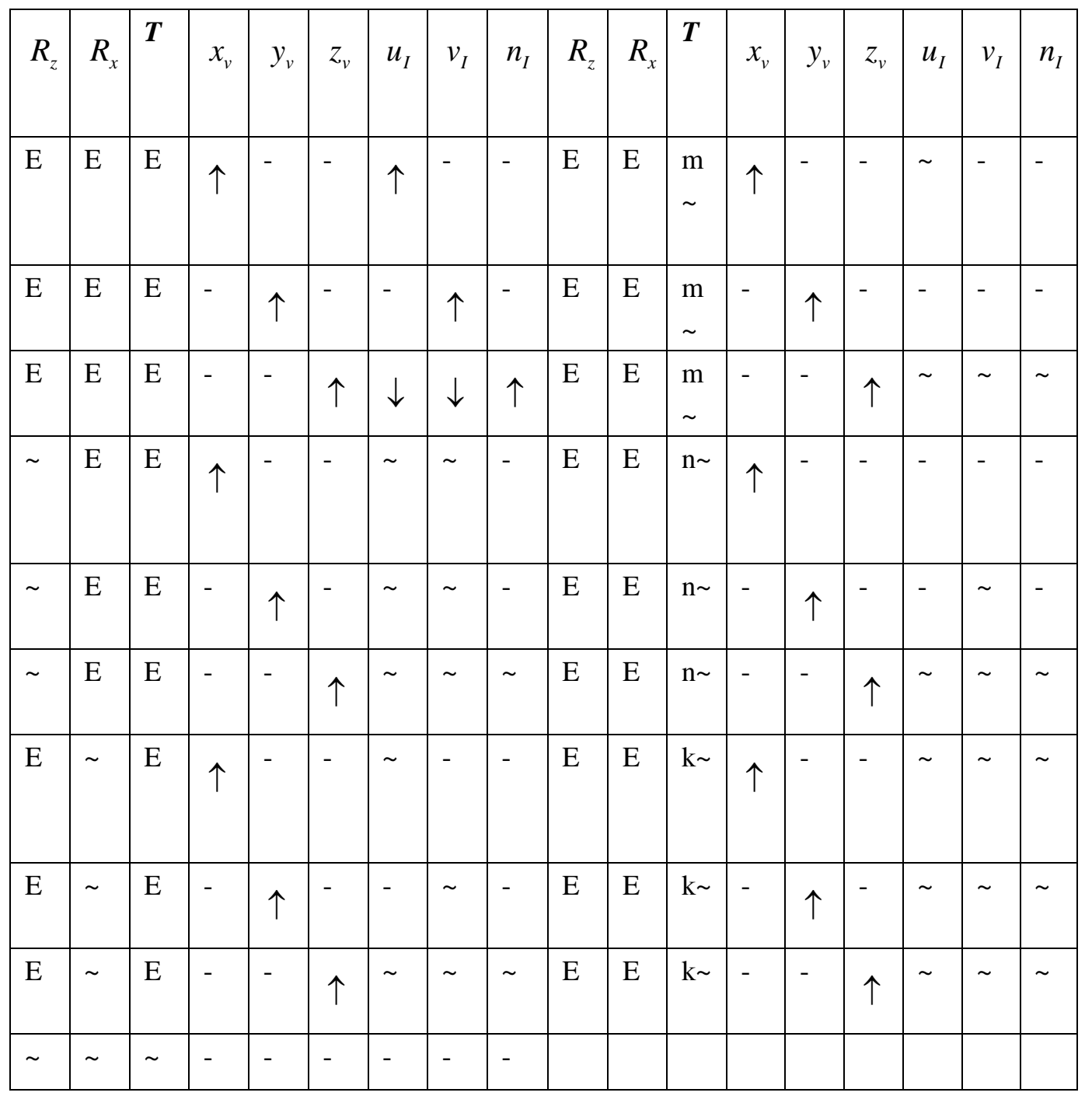

The range of view zone is about $300 \mathrm{~mm}$, and the viewing distance of is $4000 \mathrm{~mm}$. The screen size is $2000 \mathrm{~mm} \times 1125 \mathrm{~mm}$. According to the Eq. (11), the distortion factor changes with the eyes' moving. As shown in Fig.16, when the viewer is in front of the screen, the distortion factor is about zero. The red line represents the 3D area that viewers can accept. Viewers will see an incorrect and pseudoscopic image in other areas. 

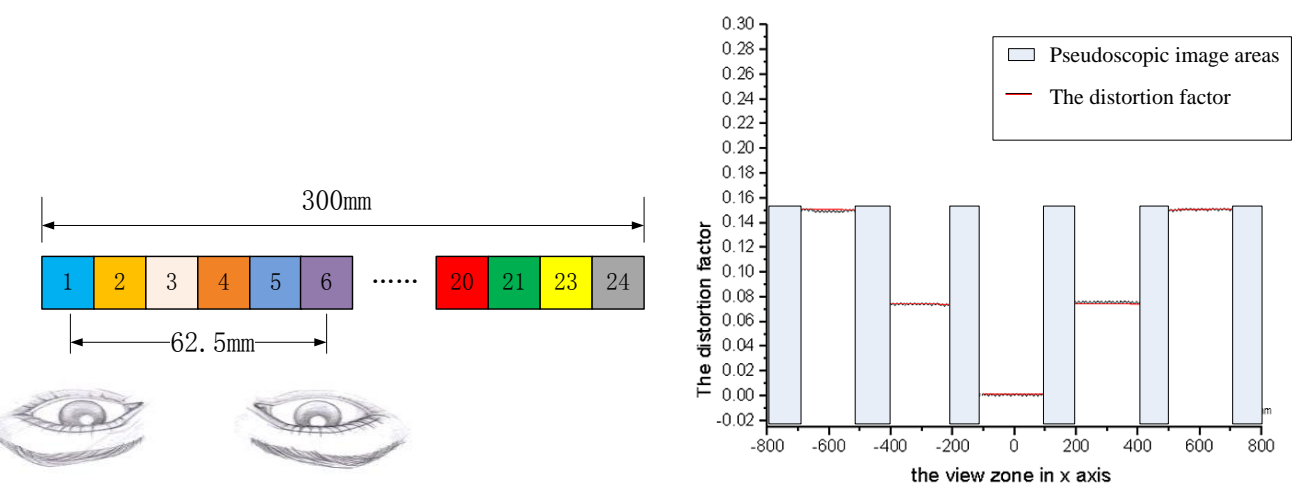

Fig.16 While the eyes are moving, the distortion factors change with eyes position which at the optimal viewing distance

Fig.17 The final results to display a 3D GIS scene that contains a airplane and a terrain map. Viewer can obtain smooth motion parallax and outstanding visual effect in a viewing zone with little distortion or no distortion.

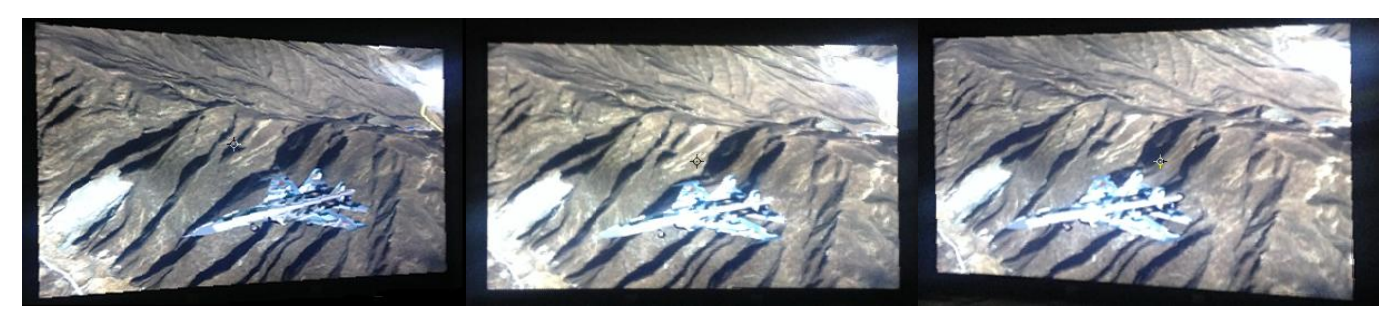

Fig.17 Terrain image and plane are shown in the 3D system and the perceived different 3D images at different position:(-600,0,4000), $(0,0,4000),(600,0,4000)$

\section{Conclusion}

In summary, a glasses-free 3D GIS projection display system with $1.9 \mathrm{~m} \times 1.2 \mathrm{~m}$ screen is demonstrated, which can synchronize and generate 24 viewpoints with 25 frame per second. The image calibration method is effective, and the distortion of geo-spatial information is mainly caused by viewers and virtual cameras' position. The relationship between the virtual camera and local geographic coordinate system can also influence the distortion of Geo-spatial information. The front-projection 3D system working with the current 3D GIS system can find many applications for viewers, such as the simulation of battlefield scene, smart city scene or Wisdom Agriculture scene.

\section{Acknowledgments:}

This work is partly supported by the "863" Program (2015AA015902), the National Science Foundation of China (61177018) and the Program of Beijing Science and Technology Plan (D121100004812001). 


\section{References:}

[1]. Zhao Dong, Su Baiquan, Chen Guowen, Liao Hongen; 360 degree viewable floating autostereoscopic display using integral photography and multiple semitransparent mirrors. Optics Express, 2015. 23(8): 9812-9823.

[2].Magalhães Daniel S F,Serra Rolando L,Vannucci André L,Moreno Alfredo B,Li Li M, Glasses-free 3D viewing systems for medical imaging. OPT LASER TECHNOL, 2012. 44(3): 650-655.

[3]Markov, V. B., Kupiec, S. A., Zakhor, A., Hooper, D., \& Saini, G. S. (2006). Autostereoscopic displays for visualization of urban environments.Proc Spie, 6392.

[4]. Bröhmer Kai, Knust Claudia, Dickmann Frank, Buchroithner Manfred F, Z-axis Based Visualization of Map Elements - Cartographic Experiences with 3D Monitors Using Lenticular Foil Technology. The Cartographic Journal, 2013. 50(3): p. 211-217.

[5]. Edler Dennis, Bestgen Anne-Kathrin, Kuchinke Lars, Dickmann Frank, True-3D Accentuating of Grids and Streets in Urban Topographic Maps Enhances Human Object Location Memory. PLOS ONE, 2015. 10(2): e0116959 1-15.

[6]. Sheat D E,Chamberlain G R,McCartney D J, Chamberlain and D.J. McCartney, Three-dimensional imaging for video telephony. Displays, 1995. 16(2): 51-54.

[7]Tianqi Zhao, Xinzhu Sang, Xunbo Yu, Xin Gao, Peng Wang, Yangdong Liu, Songlin Xie, Binbin Yan, Chongxiu Yu, and Daxiong Xu. High dense views auto-stereoscopic three-dimensional display based on frontal projection with LLA and diffused screen. Chin. Opt. Lett, 2015, 13(1):011001-1-3.

[8] Xin Gao, Xinzhu Sang, Xunbo Yu, Peng Wang, Xuemei Cao, Lei Sun, Binbin Yan, Jinhui Yuan, Kuiru Wang, Chongxiu Yu, and Wenhua Dou, Aberration analyses for improving the frontal projection three-dimensional display, Opt. Express, 2014, 22(19):23496-23511.

[9]Shujun Xing, Xunbo Yu, Tianqi Zhao, Xinzhu Sang, Yuanfa Cai, Duo Chen, Zhidong Chen, Wenhua Dou. Configuration of virtual cameras for ground based $360^{\circ}$ three-dimensional display. Optik, 2014, 125:4714-4717.

[10]. Hyung-Chul O. Li Systematic perceptual distortion of 3D slant by disconjugate eye movements. VISION RES, 2006. 46(15): 2328-2335.

[11]. Jung-Young Son,Yuri Gruts,Yuri Gruts,Joohwan Chun,Joohwan Chun,Yong-Jin Choi,Yong-Jin Choi,Jieun Bahn,Jieun Bahn,Vladimir I. Bobrinev,Vladimir I. Bobrinev, Distortion analysis in stereoscopic images. OPT ENG, 2002. 41(3): 680-685.

[12]. Dodgson N A. Variation and extrema of human interpupillary distance[J]. Proc Spie, 2004:36--46.

[13]. Chantal N. de Boer, Ria Verleur, Ard Heuvelman, Ingrid Heynderickx ., Added value of an autostereoscopic multiview 3-D display for advertising in a public environment. Displays, 2010. 31(1): $1-8$. 